\title{
Epidemiology of genital chlamydial infections in patients with chlamydial conjunctivitis; a retrospective study
}

\author{
E J Postema, L Remeijer, W I van der Meijden
}

Objective: To determine how often chlamydial conjunctivitis is accompanied by a genital chlamydial infection and if there is a correlation between the dominant hand and the eye first infected.

Methods: We retrospectively studied the records of 65 patients with chlamydial conjunctivitis who were referred to the Outpatient Department of Sexually Transmitted Diseases (STD) of the University Hospital Rotterdam by ophthalmologists of the Eye Hospital Rotterdam.

The patients have recently been asked by letter if they were left- or right-handed.

Results: Twenty of the 37 men $(54 \%)$ had a positive chlamydial urethral culture. Seventy per cent of these men had no genital symptoms. Eight of the 37 men (22\%) had a non-specific urethritis (NSU). Twenty of the 27 women examined (74\%) had a positive chlamydial cervical culture. Sixty per cent of these women had no genital symptoms. Eight women with a genital chlamydial infection also had another genital infection. Five women without a genital chlamydial infection had another genital infection. Two women had no genital infection at all. A correlation between the eye infected and left- or right-handedness of the patient could not be found.

Conclusions: A considerable percentage of the patients with a chlamydial conjunctivitis had a concomitant genital chlamydial infection. The majority of them had no genital symptoms. Since patients with chlamydial conjunctivitis and/or their partners possibly have a concomitant genital chlamydial infection, we recommend referral of both patients and sexual partners to an STD clinic for routine examination and systemic treatment when indicated.

(Genitourin Med 1996;72:203-205)

Keywords: Chlamydia trachomatis; STD; conjunctivitis

\section{Introduction}

About $0 \cdot 3-2 \%^{1-4}$ of the genital infections with Chlamydia trachomatis are complicated by a chlamydial eye infection. Inclusion conjunctivitis is the most common form of an eye infection caused by $C$ trachomatis serovars $\mathrm{D}$ $\mathrm{K}$. It normally presents as a chronic follicular conjunctivitis with mucopurulent discharge, redness and foreign-body sensation. ${ }^{56}$ This conjunctivitis is not sight threatening. It is assumed that this chlamydial conjunctivitis is the result of autoinoculation of the eye by infected genital secretion. ${ }^{4-10}$ As many patients with a chlamydial conjunctivitis have no genital symptoms, ${ }^{9-11}$ the chlamydial eye infection could be the only sign of a genital infection with $C$. trachomatis.

The complications of untreated genital chlamydial infections are serious. ${ }^{1213}$ Therefore we wanted to determine how often patients with chlamydial conjunctivitis have a concomitant genital chlamydial infection.

Department of Dermato-Venereology, University Hospital Rotterdam, Dr Molewaterplein 40, 3015 GD Rotterdam, The Netherlands E J Postema W I van der Meijden

Eye Hospital

Rotterdam

L Remeijer

Address correspondence to: Dr W I van der Meijden

Accepted for publication 15 January 1996
Transmitted Diseases (STD) of the University Hospital Rotterdam during the period of 1 October 1989 to 1 October 1994. These patients were referred by consultants of the Eye Hospital Rotterdam. All patients had a positive ocular chlamydial culture and/or immunofluorescence (IF) test. At the Outpatient STD Department urethral smears were taken in male patients. In female patients samples from the cervix were taken.

The 65 patients have recently been asked by letter if they would let us know whether they were left- or right-handed.

\section{Results}

During the period mentioned 88 adults and adolescents with a chlamydial ocular infection visited the Eye Hospital Rotterdam. Sixty-five of them $(74 \%)$ also visited the Outpatient STD Department. Thirty-seven of the 65 patients $(57 \%)$ were men, $28(43 \%)$ were women. The age of the male patients was 18-56 years (mean 27.4; median 24.2). The age of the female patients was $15-51$ years (mean 23.4; median 20.0).

Fifty-four per cent (20/37) of the men with a chlamydial conjunctivitis had a positive chlamydial urethral culture. Six of these 20 men $(30 \%)$ had genital complaints, such as dysuria and/or urethral discharge. Fourteen (70\%) had no genital complaints. Eight of the 17 men without a positive chlamydial urethral sample, ie $22 \%(8 / 37)$ of all men had a non-
We retrospectively studied the records of all 65 patients with chlamydial conjunctivitis who visited the Outpatient Department of Sexually 
Table 1 Current STD diagnoses among women

\begin{tabular}{lll}
\hline & $\begin{array}{l}\text { Genital } \\
\text { chlamydial infection }\end{array}$ & $\begin{array}{l}\text { No genital } \\
\text { chlamydial infection }\end{array}$ \\
\hline Gonorrhoea & $1(5 \%)$ & - \\
Condylomata acuminata & $2(10 \%)$ & $1(14 \%)$ \\
Trichomoniasis & $1(5 \%)$ & $1(14 \%)$ \\
Vaginal candidosis & $1(5 \%)$ & $2(29 \%)$ \\
Bacterial vaginosis (BV) & $3(15 \%)$ & $1(14 \%)$ \\
Trichomoniasis and BV & $-12(60 \%)$ & $2(29 \%)$ \\
No (concomitant) genital infection & 20 & 7 \\
Total & & \\
\hline
\end{tabular}

Table 2 Correlation between the eye infected with $C$ trachomatis and left-or righthandedness of the patient

\begin{tabular}{lcl}
\hline & Right-handed & Left-handed \\
\hline Unilateral conjunctivitis right & $8(27 \%)$ & $2(40 \%)$ \\
Unilateral conjunctivitis left & $6(20 \%)$ & $2(40 \%)$ \\
Bilateral conjunctivitis started right & $3(10 \%)$ & - \\
Bilateral conjunctivitis started left & $5(17 \%)$ & $1(20 \%)$ \\
Bilateral conjunctivitis not specified & $8(27 \%)$ & - \\
Total & 30 & 5 \\
\hline
\end{tabular}

specific urethritis (NSU). The men examined had no concomitant other genital infections.

One of the 28 women refused to allow genital samples to be taken. Seventy-four per cent $(20 / 27)$ of the remaining women had a positive chlamydial cervical culture. Seven of these 20 women $(35 \%)$ had genital complaints, such as vaginal discharge. Twelve $(60 \%)$ had no genital complaints. One foreign woman could not tell if she had genital complaints. Eight women with a genital chlamydial infection had a concomitant genital infection (table 1). Five women without a genital chlamydial infection had another genital infection (table 1). Two of the 27 women examined ( $7 \%$ ) had no genital infection at all.

It took 2-283 days (mean 25; median 16) after the first visit at the Eye Hospital before the patients visited the STD Department for the first time. The mean period between first Eye Hospital visit and first STD Department visit was 23 days (median: 13 days) for those with positive genital samples, and 29 days (median: 20 days) for those with negative genital samples for $C$ trachomatis.

Twenty-four of the 65 patients (37\%) had a unilateral conjunctivitis of the right eye, 13 $(20 \%)$ a unilateral conjunctivitis of the left eye, $27(42 \%)$ a bilateral conjunctivitis, and in one case $(2 \%)$ it was unknown which eye was infected. Nineteen per cent $(5 / 27)$ of the bilateral eye infections started in the right eye, 30\% $(8 / 27)$ in the left eye, and in $52 \%(14 / 27)$ it was not known in which eye the bilateral conjunctivitis had started.

Thirty-five patients answered the question about being left- or right-handed. The results of the inquiry are listed in table 2 .

\section{Discussion}

A large proportion of the men and women with a chlamydial conjunctivitis $(54 \%$ and $74 \%$, respectively) had a concomitant genital chlamydial infection. Other studies have found genital chlamydial infection rates of $45-61 \%$ in $\operatorname{men}^{49.1114}$ and $56-90 \%$ in women. ${ }^{3491114}$ Although we assumed that the eye infection was the result of autoinoculation with infected genital secretion, a genital infection with $C$ trachomatis could not be proven in 38\% (24/64) of our patients.

There are some possible explanations for this discrepancy. Stenberg and Mårdh suggest that the genital infection may heal before the eye infection begins. ${ }^{4}$ We found a mean delay of over two weeks between the first visit to the Eye Hospital and the first visit to the STD Department. There was also a difference between the mean time taken to attend the STD Department for those with positive versus those with negative genital chlamydial samples. Both findings contribute to the relevance of Stenberg's hypothesis.

Another explanation might be the use of antibiotics prior to genital sampling. Two men and two women had been given a prescription for doxycycline by the ophthalmologist prior to their visit at the STD Department. The genital samples taken appeared to be negative for $C$ trachomatis.

Sexual partners of patients with chlamydial conjunctivitis might be infected genitally, without the patients themselves being infected genitally. ${ }^{14}$ There could be transmission of infected genital secretion of the partner to the patient's eye. Eye-to-eye infections (with infectious material from the eyes of partners or relatives) have been described. ${ }^{35714}$

Since chlamydial culture techniques and IF tests were used instead of very sensitive techniques like polymerase chain reaction (PCR), the possibility of false negative genital samples cannot be excluded. Finally, we may not exclude the possibility of false positive conjunctival samples, although this is very unlikely.

The hand probably plays a crucial part in the transmission of $C$ trachomatis to the eye. Neither we nor Stenberg and $M^{2} a^{2} d^{4}$ were able to find a correlation between the dominant hand and eye infection on the ipsilateral side, as reported by Insler et al. ${ }^{8}$

Considering the strong association between chlamydial eye and genital infections, any physician treating a patient with a chlamydial conjunctivitis should consider the probability of a genital infection in this patient and/or in the sexual partner(s). Especially the general practitioner and ophthalmologist ${ }^{1516}$ are important in this respect. We recommend referral of patients (and sexual partners) to an STD Clinic for investigation and treatment of chlamydial and other genital infections. ${ }^{10} 17$ Treatment of genital and ocular chlamydial infections should be systemic. ${ }^{37151718}$ In our STD Department treatment (of non-pregnant patients) consisted of doxycycline $100 \mathrm{mg}$ twice a day for 7 days. At present treatment consists of azithromycin 1 gram as a single dose. Pregnant women are treated with erythromycin $500 \mathrm{mg}$ four times a day for 7 days. Eye symptoms can be treated locally with tetracycline ointment, but also react well to systemic treatment. 
We thank Dr J Buitenwerf and Dr AM Dingemans-Dumas for supplying data and for their useful comments on the manuscript.

1 Tullo AB, Richmond SJ, Easty DL. The presentation and incidence of paratrachoma in adults. $\mathcal{f}$ Hygiene (Lond) 1981;87:63-9.

2 Møller BR, Postgonococcal conjunctivitis caused by Chlamydia trachomatis. Case report. $\mathrm{Br} f$ Venereal Dis 1983;59:109-10

3 Rönnerstam R, Persson K, Hansson H, Renmarker K. Prevalence of chlamydial eye infection in patients attending an eye clinic, a VD clinic, and in healthy persons. Br Opthalmol 1985;69:385-8.

4 Stenberg K, Mårdh P-A. Genital infection with Chlamydia trachomatis in patients with chlamydial conjunctivitis: unexplained results. Sex Transm Dis 1991;18:1-4.

5 Jones BR. Ocular syndromes of TRIC virus infection and their possible genital significance. $\mathrm{Br} \mathcal{F}$ Venereal Dis 1964;40:3-15.

6 Viswalingam ND, Wishart MS, Woodland RM. Adult chlamydial ophthalmia (paratrachoma). $\mathrm{Br}$ Med Bull 1983;39:123-7.

7 Rönnerstam R, Persson K. Chlamydial eye infection in adults. Scand F Infect Dis Suppl 1982;32:111-5.

8 Insler MS, Anderson AB, Murray M. Latent oculogenital infection with Chlamydia trachomatis. Ophthalmology

9 Patel HC, Viswalingam ND, Goh BT. Chlamydial ocular infection: efficacy of partner notification by patien referral. Int $\mathcal{F} S T D$ AIDS 1994;5:244-7.
10 Garland SM, Malatt A, Tabrizi S, et al. Chlamydia trachomatis conjunctivitis. Prevalence and association with gential tract infection. Med $\mathcal{f}$ Aust 1995;162:363-6.

11 Stenberg K, Mårdh P-A. Chlamydial conjunctivitis in neonates and adults. History, clinical findings and followup. Acta Ophthalmol (Copenh) 1990;68:651-7.

12 Luger A. Epidemiologie, Klinik und Therapie der Infektionen mit Chlamydia trachomatis Serotyp D-K. Wien Klin Wochenschr 1987;99:1-14.

13 Sweet RL, Schachter J, Landers DV. Chlamydial infections in obstetrics and gynecology. Clin Obstet Gynecol 1983, in obstetrics

14 Vaughan-Jackson JD, Dunlop EMC, Darougar S, Dwyer RSC, Jones BR. Chlamydial infection. Results of tests for Chlamydia in patients suffering from acute Reiter's disease compared with results of tests of the genital tract and rectum in patients with ocular infection due to TRIC agent. Br F Venereal Dis 1972;48:445-51.

15 Belloir-Furet F. Le rôle de l'ophtalmologiste dans le dépistage des maladies sexuellement transmissibles à Chlamydia trachomatis - a propos de 194 cas. Bull Soc Ophtalmol Fr 1989;89:403-12.

16 Janßen KT, Siemon P, Bialasiewicz AA. Okuläre Chlamydieninfektionen im Münsterland. Eine klinische Studie an 409 Patienten. Ophthalmologe 1994;91: 671-5.

17 Darougar S, Viswalingam ND. Marginal corneal abscess associated with adult chlamydial ophthalmia. $\mathrm{Br} f$ Ophthalmol 1988;72:774-7.

18 Editorial. Chlamydial infections of the eye. Lancet 1977;ii 857-8. 